\title{
Relaxation and Transition of Copolymers of Vinylidene Fluoride and Trifluoroethylene with High Trifluoroethylene Content
}

\author{
Yukinobu MurATA \\ Osaka Prefectural Technical College, 26-12, Saiwai-cho, \\ Neyagawa, Osaka 572, Japan \\ (Received February 10, 1988)
}

\begin{abstract}
Dielectric behavior and related properties were investigated for copolymers of vinylidene fluoride (VDF) and trifluoroethylene (TrFE) with TrFE content from 35 to $87 \mathrm{~mol} \%$. Three dielectric loss peaks, designated as $\beta^{\prime}, \beta$, and $T_{\mathrm{t}}$ were observed in increasing order of temperature. The $\beta^{\prime}$ relaxation was predominant in samples with high crystallinity for all copolymers and was connected with molecular motion around defects due to anomalous linkages of head-to-head and tail-to-tail of TrFE rich sequences. The $\beta$ relaxation found in samples with low crystallinity was related to micro-Brownian motion of VDF rich sequences in main chains for 35 to $67 \mathrm{~mol} \%$ TrFE copolymers. In copolymers with 71 and $87 \mathrm{~mol} \% \mathrm{TrFE}$, the $\beta$ relaxation was considered to consist of two components, $\beta_{\mathrm{a}}$ and $\beta_{\mathrm{c}}$, due to the local molecular motions in amorphous and crystalline regions, respectively. The $T_{\mathrm{t}}$ loss peak was related to a transition between the phase of antiferroelectric nature and paraelectric phase for 48 to $87 \mathrm{~mol} \% \operatorname{TrFE}$ copolymers. The transition for 63 and $71 \mathrm{~mol} \%$ TrFE copolymers exhibited characters of the first or second order of the transition, depending on the physical properties observed. Pressure dependence of the $T_{\mathrm{t}}$ transition temperature was smaller for 63 and $71 \mathrm{~mol} \%$ TrFE copolymers than that for 48 and $35 \mathrm{~mol} \%$ TrFE copolymers.

KEY WORDS Vinylidene Fluoride-Trifluoroethylene Copolymer / High Trifluoroethylene Content / Dielectric Relaxation / Defect / Head-to-Head / Tail-to-Tail / Phase Transition /
\end{abstract}

The relaxation behavior of copolymers of vinylidene fluoride (VDF) and trifluoroethylene (TrFE) with high TrFE content was studied by dynamic mechanical measurements. $\alpha$ and $\beta$ relaxations due to micro-Brownian motion of TrFE and VDF rich sequences were found at 313 and $250 \mathrm{~K}$, respectively, for copolymers with 48 to $71 \mathrm{~mol} \%$ TrFE. ${ }^{1}$ Yagi also found two relaxations connected with micro-Brownian motion for copolymers with 40 to $63 \mathrm{~mol} \%$ TrFE in dynamic mechanical study. $^{2}$ Furukawa et al. ${ }^{3}$ studied dielectric behavior of VDF-TrFE copolymers with the entire range of comonomer content and found a relaxation at $253 \mathrm{~K}$ at $1 \mathrm{kHz}$. The relaxation at $253 \mathrm{~K}$ was ascribed to the same type of molecular motion in the amorphous region as that of the transformation of trans and gauche at the ferroelectric to paraelectric transition. Koizumi et al. ${ }^{4}$ found $\beta$ relaxation due to micro-Brownian motion around $250 \mathrm{~K}$ and $\gamma$ relaxation connected with local molecular motion in frozen glassy state at lower temperature. Different relaxation mechanisms were presented for relaxation around $253 \mathrm{~K}$ by these authors. For polytrifluoroethylene (PTrFE) two types of relaxation in the crystalline region were found below room temperature and considered to be due to reorientation of main chains around row vacancy defects at chain ends and molecular motion of disordered chains around anomalous linkages such as 
head-to-head and tail-to-tail in crystals. ${ }^{5}$ Such motion of main chains may take place also in copolymers with TrFE rich content since TrFE rich sequences exist in these copolymers.

It is well known that VDF-TrFE copolymers with $65-80 \mathrm{~mol} \%$ VDF adopt the form I crystal of poly(vinylidene fluoride) (PVDF) ${ }^{6,7}$ and exhibit the ferroelectric to paraelectric transition with the peak ${ }^{4,8}$ of dielectric constant and a dielectric displacement $D$ vs. electric field $E$ hysteresis of rectangular shape. ${ }^{9.10}$ The transition has the nature of the first order of transition since pressure dependence of the transition temperature is expressed by the Clausius-Clapayron equation and since thermal hysteresis of the transition temperature is observed. ${ }^{4}$ In contrast to copolymers with high VDF content, copolymers with high TrFE content and even PTrFE showed two current peaks in polarization current density $j$ vs. $E$ curves which corresponded to the double hysteresis in $D-E$ curves. X-Ray measurements under high electric field revealed that the two current peaks were related to a phase transition between two slightly different crystal structures. ${ }^{11-13}$ These structures were characterized by double and single peaks around $18-20^{\circ}$ in X-ray diffraction angle $2 \theta$ and were referred to as $\mathrm{D}$ and $\mathrm{S}$ phases, respectively. It was inferred from density and remanent polarization data that molecular chains with $T_{3} G_{3} \bar{G}$ conformation were packed antiparallel and parallel for the $\mathrm{D}$ and $\mathrm{S}$ phases, respectively. ${ }^{12,13}$

In this paper, dielectric relaxation behavior was examined to reveal molecular mechanism for VDF-TrFE copolymer samples of 35 to $87 \mathrm{~mol} \%$ TrFE with different crystallinity and is discussed in comparison with the relaxation mechanism of PTrFE. The dielectric behavior and thermal and linear expansion properties at the $T_{\mathrm{t}}$ transition were studied for TrFE rich copolymers and compared with those for VDF rich copolymers. Pressure dependence of the transition temperature was also investigated for the TrFE rich copolymers.

\section{EXPERIMENTAL}

The samples used were VDF-TrFE copolymers with 35 to $87 \mathrm{~mol} \%$ TrFE and supplied by Daikin Industries Ltd. These samples were heat-pressed at $523 \mathrm{~K}$ into films with 30 $100 \mu \mathrm{m}$ in thickness. To obtain specimens with different crystallinity, two cooling conditions the same as those in the previous work ${ }^{1}$ were used. X-Ray diffraction measurements were performed by a Rigaku Denki diffractometer Geigerflex 2012. The crystallinities estimated from X-ray diffraction curves from 5 to $35^{\circ}$ in diffraction angle $2 \theta$ and the density measured by the floating method are listed in Table I. Thermal properties were observed by a Rigaku Denki differential thermal analyzer Thermoflex TG-DTA. Dielectric measurements were carried out in a temperature range from 193 to $373 \mathrm{~K}$ and over a frequency range from $1 \mathrm{~Hz}$ to $1 \mathrm{MHz}$, using an Ando Denki transformer bridge Model TR-1C and an operational amplifier bridge. Dielectric measurements under hydrostatic pressure were made using a three-terminal electrode immersed in a high pressure vessel. ${ }^{4}$ Polarization current density $j v s$. electric field $E$ and dielectric displacement $D v s$. electric field $E$ curves were obtained using a high-voltage triangular wave at $0.01 \mathrm{~Hz}$ by methods described elsewhere. ${ }^{11}$

Table I. Crystallinity and density of samples

\begin{tabular}{|c|c|c|c|}
\hline \multirow{2}{*}{$\begin{array}{c}\text { Sample } \\
\text { No. }\end{array}$} & TrFE content & Crystallinity & Density \\
\hline & $\mathrm{mol}^{\circ} \%$ & $\%$ & $10^{3} \mathrm{~kg} \mathrm{~m}^{-3}$ \\
\hline $1^{\mathrm{a}}$ & 35 & 32 & 1.856 \\
\hline $2^{b}$ & 35 & 45 & 1.911 \\
\hline $3^{\mathbf{a}}$ & 48 & 29 & 1.886 \\
\hline $4^{b}$ & 48 & 48 & 1.916 \\
\hline $5^{\mathrm{a}}$ & 63 & 26 & 1.888 \\
\hline $6^{\mathrm{b}}$ & 63 & 43 & 1.940 \\
\hline $7^{\mathbf{a}}$ & 71 & 31 & 1.928 \\
\hline $8^{b}$ & 71 & 39 & 1.959 \\
\hline $9^{a}$ & 87 & 26 & 1.934 \\
\hline $10^{\mathrm{b}}$ & 87 & 41 & 1.981 \\
\hline
\end{tabular}

a Quenched.

b Slowly cooled. 
Sample films about $30-80 \mu \mathrm{m}$ thick with evaporated aluminum electrodes were poled for X-ray measurements using the triangular wave field. Thermal expansion data were taken on molded rod specimens of $6 \mathrm{~mm}$ diameter and $4 \mathrm{~mm}$ long by a displacement transducer immersed in silicone oil. ${ }^{14}$

\section{RESULTS AND DISCUSSION}

\section{Low Temperature Relaxations}

Plots of dielectric loss $\varepsilon^{\prime \prime} v s$. temperature $T$ in quenched and slowly cooled samples (5 and 6) of $63 \mathrm{~mol} \% \mathrm{TrFE}$ copolymer are shown in Figures 1 and 2, respectively. Two prominent loss peaks were observed at 250-290 and $338 \mathrm{~K}$ in these samples and a small shoulder was at $320 \mathrm{~K}$ in slowly cooled sample 6 . In the previous mechanical study, ${ }^{1}$ the $T_{\mathrm{t}}$ transition and $\alpha$ and $\beta$ relaxations were found for this copolymer in decreasing order of temperature. The $T$, transition was ascribed to the ferroelectric to paraelectric phase transition and $\alpha$ and $\beta$ relaxations were considered due to micro-Brownian motion of TrFE and VDF rich sequences, respectively. A comparison of dielectric and mechanical results indicated the dielectric loss peaks at 338 and $250-290 \mathrm{~K}$ to correspond to the $T_{\mathrm{t}}$ transition and $\beta$ relaxation, respectively and the shoulder to be related to the $\alpha$ relaxation. In the quenched sample, no $\alpha$ relaxation was observed and $\varepsilon^{\prime \prime}$ increased at a low frequency of 10 and $100 \mathrm{~Hz}$ with increasing temperature above $300 \mathrm{~K}$. The increase of $\varepsilon^{\prime \prime}$ was similar to that above the glass transition in PTrFE, being ascribed to the increase of dc conductance at higher temperature. ${ }^{15}$ Thus, the $\alpha$ loss peak was concealed by a large value of $\varepsilon^{\prime \prime}$ due to dc conductance.

The details of the $\beta$ relaxation for $63 \mathrm{~mol} \%$ TrFE copolymer (samples 5 and 6) are demonstrated as contour diagrams of $\varepsilon^{\prime \prime}$ against frequency $f$ and reciprocal of temperature $1 / T$ in Figures 3 and 4. For the quenched sample 5, the $\beta$ relaxation was observed as a prominent mountain ridge. As the temperature was low-

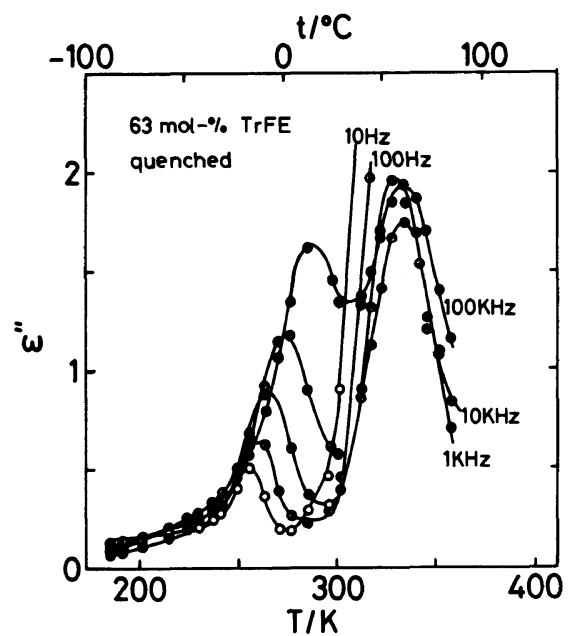

Figure 1. Temperature dependence of dielectric loss $\varepsilon^{\prime \prime}$ for quenched sample with $63 \mathrm{~mol} \%$ TrFE (sample 5).

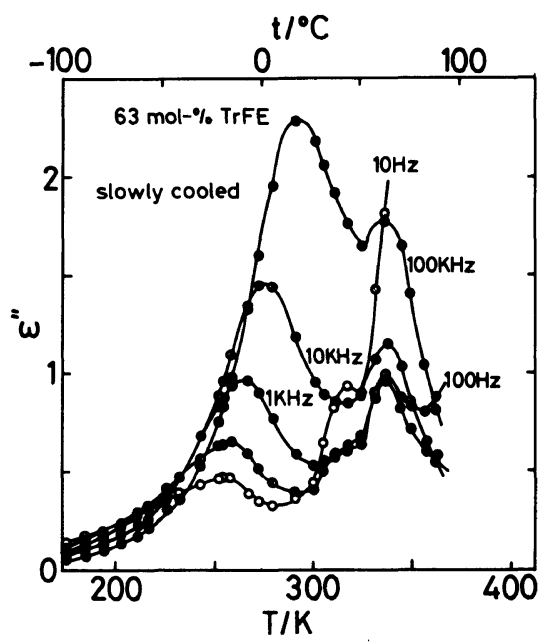

Figure 2. The same as Figure 1 for slowly cooled sample with $63 \mathrm{~mol} \%$ TrFE (sample 6).

ered, a small ridge designated as $\beta^{\prime}$ branched off from the $\beta$ relaxation. The dotted line shows the position of frequency $f_{\mathrm{M}}$ at the loss peak in frequency dispersion and the dotted dashed line shows the position of temperature at the loss peak in temperature dispersion. The temperature dependence of $f_{\mathrm{M}}$ was found to be the WLF type for the $\beta$ relaxation and to be the Arrhenius type for the $\beta^{\prime}$ relaxation. For slowly cooled sample 6 , the mountain ridge 


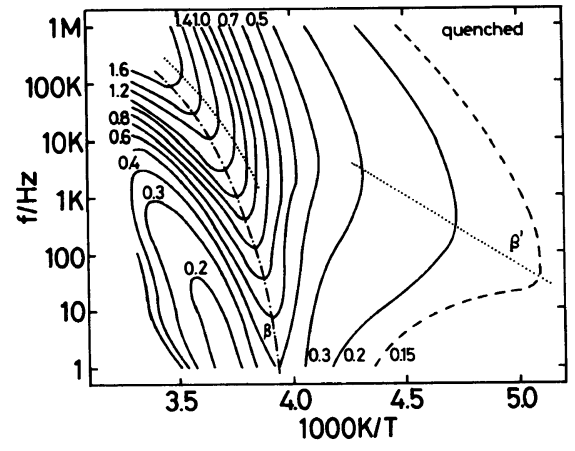

Figure 3. Contour diagram of $\varepsilon^{\prime \prime}$ as a function of frequency and reciprocal of temperature in a temperature region below room temperature for quenched sample with $63 \mathrm{~mol} \%$ TrFE (sample 5).

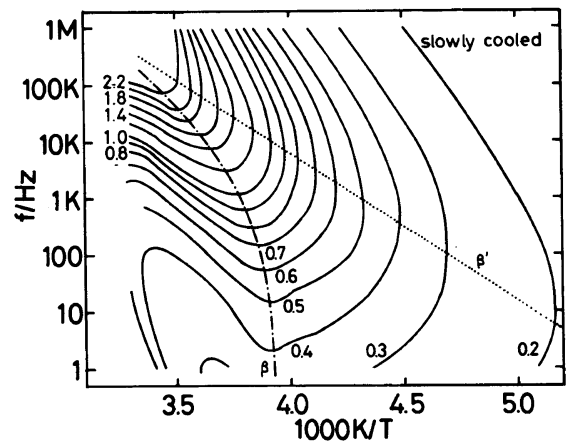

Figure 4. The same as Figure 3 for slowly cooled sample with $63 \mathrm{~mol} \%$ TrFE (sample 6).

became broad and only one relaxation process was found in the $f_{\mathrm{M}} v s$. $1 / T$ curve, owing to increase in the intensity of the $\beta^{\prime}$ relaxation. The relaxation intensity $\Delta \varepsilon$ in a temperature range below room temperature $\mathrm{RT}$ was estimated from the frequency dependence of the dielectric constant and loss, $\varepsilon^{\prime}$ and $\varepsilon^{\prime \prime}$, fitted by the Cole-Cole equation and plotted against temperature for samples 5 and 6 in Figure 5. The values of $\Delta \varepsilon$ increased abruptly around $250 \mathrm{~K}$ for quenched sample 5 and steadily for slowly cooled sample 6 . The WLF type temperature dependence of $f_{\mathrm{M}}$ and abrupt increase of $\Delta \varepsilon$ for the $\beta$ relaxation of quenched sample. 5 indicate that the $\beta$ relaxation is due to microBrownian motion of the main chains in the amorphous region. The temperature of $250 \mathrm{~K}$

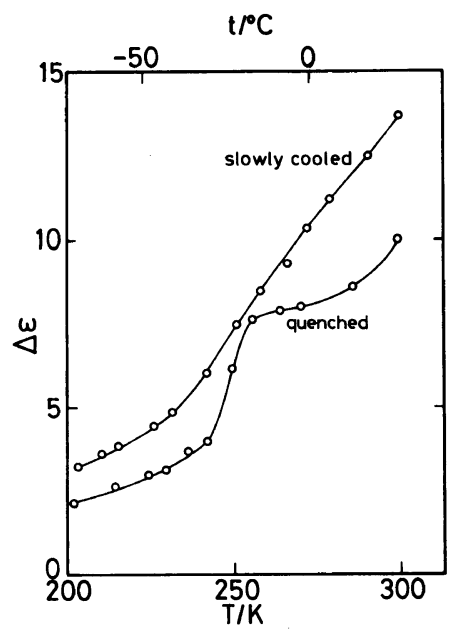

Figure 5. Temperature dependence of relaxation strength $\Delta \varepsilon$ for quenched and slowly cooled samples with $63 \mathrm{~mol} \%$ TrFE (samples 5 and 6 ).

for the abrupt increase of $\Delta \varepsilon$ corresponds to the glass transition. ${ }^{1}$ On the contrary, the $\beta^{\prime}$ relaxation of slowly cooled sample 6 was characterized by cotinuous increase in $\Delta \varepsilon$ with temperature and the Arrhenius type temperature dependence of $f_{\mathrm{M}}$. The values of $\Delta \varepsilon$ in the temperature range below RT were larger for slowly cooled sample 6 with high crystallinity than those for quenched sample 5 with low crystallinity. Thus, the $\beta^{\prime}$ relaxation is connected with molecular motion in the crystalline region.

Temperature dependence of $\varepsilon^{\prime \prime}$ at $10 \mathrm{~Hz}$ is shown for all quenched and slowly cooled samples with 35 to $87 \mathrm{~mol} \%$ TrFE in Figure 6. The shape of the loss peak is sharp in quenched samples with 35 and $48 \mathrm{~mol} \%$ TrFE (samples 1 and 3) and becomes broad in samples with 71 to $87 \mathrm{~mol} \%$ TrFE (samples 7 and 9) while it does not vary with TrFE content in slowly cooled samples. Variation of $f_{\mathrm{M}}$ with the reciprocal of temperature $1 / T$ is shown for quenched samples of 35 to $87 \mathrm{~mol} \%$ TrFE copolymers in Figure 7 and for slowly cooled samples in Figure 8. In quenched samples, the $\beta$ relaxation of the WLF type at high temperature and the $\beta^{\prime}$ relaxation of the Arrhenius 


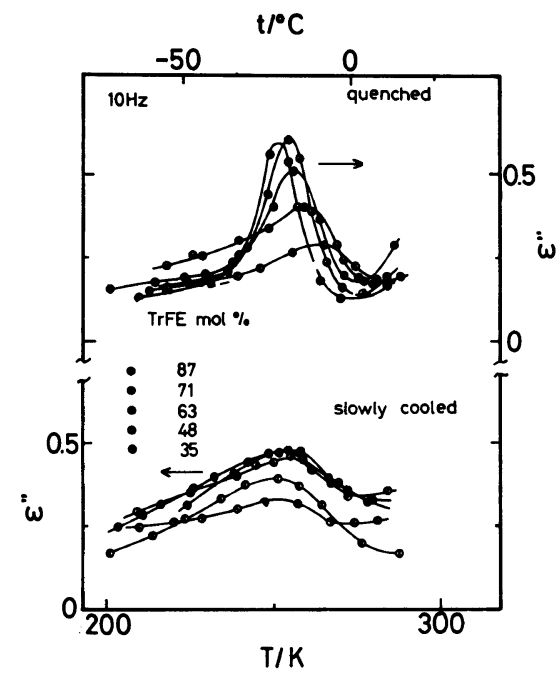

Figure 6. Temperature dependence of $\varepsilon^{\prime \prime}$ at $10 \mathrm{~Hz}$ in quenched (upper) and slowly cooled (lower) samples for 35 to $87 \mathrm{~mol} \%$ TrFE copolymers.

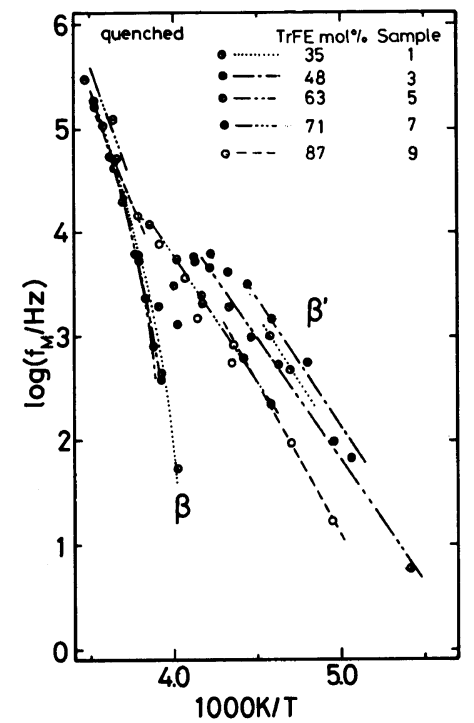

Figure 7. Plots of relaxation frequency $f_{\mathrm{M}}$ against reciprocal of temperature for quenched samples for 35 to $87 \mathrm{~mol} \%$ TrFE copolymers.

type at low temperature were found in samples 1 and 3 to be essentially the same as in sample 5 (Figure 3), while two relaxations of the Arrhenius type were observed for samples 7 and 9. In slowly cooled samples, the $\beta^{\prime}$ relaxation of the Arrhenius type as observed for

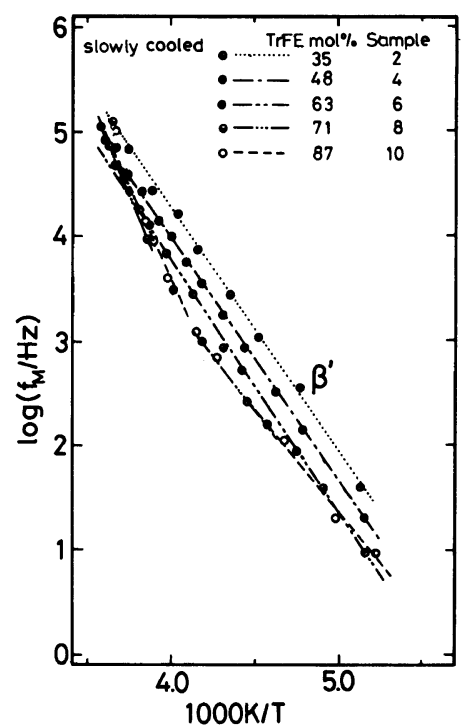

Figure 8. The same as Figure 7 in slowly cooled samples.

$63 \mathrm{~mol} \%$ TrFE copolymer (sample 6) in Figure 4 was found in 35 and $48 \mathrm{~mol} \%$ TrFE copolymers (samples 2 and 4) while 71 and $87 \mathrm{~mol} \%$ TrFE copolymers (samples 8 and 10) exhibited two relaxations of the Arrhenius type. The values of $\Delta \varepsilon$ were obtained mostly by the Cole-Cole equation although the $\beta$ relaxation for samples 1 and 3 was approximated by the Williams-Watts equation. ${ }^{16}$ In quenched samples, the abrupt increase of $\Delta \varepsilon$ took place in samples 1 and 3 in the same manner as that for sample 5 and the gradual increase was found for samples 7 and 9 as shown in Figure 9. For slowly cooled samples, $\Delta \varepsilon$ increased steadily for all samples in Figure 10. In the previous mechanical study, ${ }^{1} \alpha$ and $\beta$ relaxations were found at $300-313 \mathrm{~K}$ and $250 \mathrm{~K}$ and ascribed to micro-Brownian motion of TrFE rich and VDF rich sequences, respectively. Thus, this relaxation mechanism is confirmed for the dielectric $\beta$ relaxation of quenched samples 1 , 3 , and 5. Quenched sample 7 gives a somewhat broader loss peak in Figure 6 since the crystallinity is higher for this sample than those for other quenched samples and since VDF rich sequences may be less than samples 1 and 3 as 


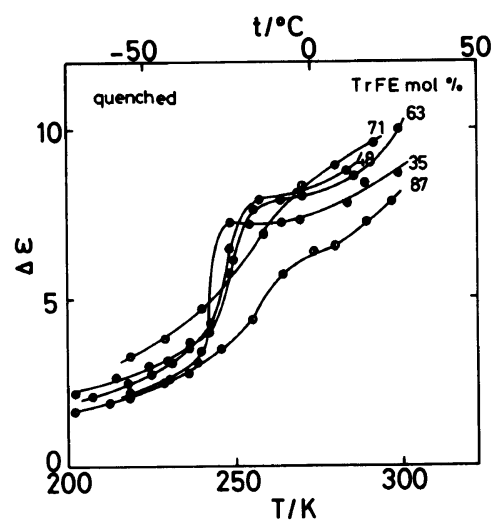

Figure 9. Temperature dependence of $\Delta \varepsilon$ for quenched samples with 35 to $87 \mathrm{~mol} \%$ TrFE.

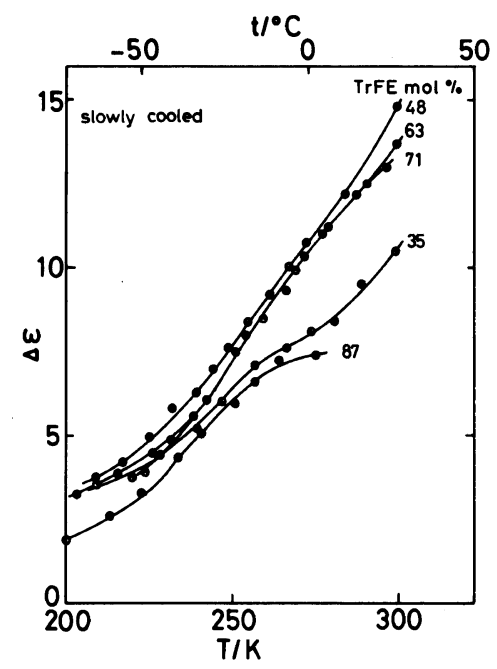

Figure 10. The same as Figure 9 for slowly cooled samples.

expected from low VDF content. No sharp loss peak was observed for quenched sample 9 . It seems that there are no VDF rich sequences at this content so that similar relaxations to those in $\mathrm{PTrFE}^{5}$ take place.

Slowly cooled samples 2 and 4 show the broad loss peak, gradual increase of $\Delta \varepsilon$ and the Arrhenius type of temperature dependence of $f_{\mathrm{M}}$ which are similar to those of the $\beta^{\prime}$ relaxation for sample 6. The values of $\Delta \varepsilon$ are larger for slowly cooled samples than those for quenched samples. Thus the $\beta^{\prime}$ relaxation in the crystalline region is predominant also in these samples. An activation energy of the $\beta^{\prime}$ relaxation is similar to that of the $\gamma$ relaxation for PVDF. ${ }^{17}$ Koizumi et al. ${ }^{4}$ studied copolymers with TrFE content lower than $48 \mathrm{~mol} \%$ by the dielectric method. They found the $\gamma$ relaxation corresponding to the $\beta^{\prime}$ relaxation in this study and ascribed the relaxation to the local molecular motions in the frozen glassy state. The temperature dependence of $f_{M}$ in slowly cooled samples 8 and 10 was different from that for other slowly cooled samples. Two relaxations of the Arrhenius type were observed for samples 8 and 10 in contrast to one relaxation process for samples 2,4 , and 6 . Temperature dependence of $f_{M}$ for samples 8 and 10 is similar to that of PTrFE in which $\beta$ and $\beta^{\prime}$ relaxations of the Arrhenus type were observed in the temperature region below RT. The $\beta$ relaxation at high temperature consisted of two components $\beta_{\mathrm{a}}$ and $\beta_{\mathrm{c}}$ which were ascribed to the local mode of molecular motion in the amorphous region and to reorientation of main chains around the chain end defects in the crystals. ${ }^{5}$ The values of $\Delta \varepsilon$ for the $\beta$ and $\beta^{\prime}$ relaxations are also larger for slowly cooled samples 8 and 10 than those for quench ed samples 7 and 9 as shown in Figures 9 and 10 . Thus, the $\beta_{\mathrm{c}}$ and $\beta^{\prime}$ relaxations assigned to the crystalline region are predominant in slowly cooled samples 8 and 10 . The latter relaxation took place in all slowly cooled samples of 35 to $87 \mathrm{~mol} \%$ TrFE copolymers. The activation energy of the $\beta^{\prime}$ relaxation for these copolymers was $38-47 \mathrm{~kJ} \mathrm{~mol}^{-1}$ and consistent with that of the $\beta^{\prime}$ relaxation for $\mathrm{PTrFE}^{5}$ which was related to molecular motion of the main chains of TrFE sequences with irregular and disordered conformations around the defects of anomalous linkages such as head-to-head and tail-to-tail in crystals. Thus, it was inferred that copolymers with 48 to $87 \mathrm{~mol} \%$ TrFE contain TrFE rich sequences. ${ }^{1}$ TrFE rich sequences are likely contained even in $35 \mathrm{~mol} \%$ TrFE copolymer since the mechanical $\alpha$ relaxation was found in this copolymer although the relaxation intensity 
was weak..$^{18}$ It seems that TrFE rich sequences yield the same type of defects in crystals for these copolymers as that for PTrFE. The main chains around the defects possibly take on disordered and irregular conformations. It is likely that the $\beta^{\prime}$ relaxation is due to molecular motion of these molecular chains with disordered and irregular conformation in crystals.

Furukawa et al. ${ }^{3}$ studied the same copolymers as those used in this work by dielectric measurements. They. observed a relaxation around $253 \mathrm{~K}$ at $1 \mathrm{kHz}$ and assigned it to the molecular motion in the amorphous region. In their results, temperature dependence of the relaxation time for the relaxation was of the Arrhenius type and the activation energy was the same as that for the relaxation at the ferroelectric to paraelectric transition. From these results they considered that the relaxation at $250 \mathrm{~K}$ at $1 \mathrm{kHz}$ was related to the same type of molecular motion in the amorphous region as that at the ferroelectric to paraelectric transition. However, the relaxation behavior is similar to that of the $\beta^{\prime}$ relaxation in this study.

Variation of $\Delta \varepsilon$ with TrFE content in the $\beta^{\prime}$ relaxation is shown for slowly cooled samples in Figure 11, where $\Delta \varepsilon$ increases with decreasing TrFE content, showing a maximum at about $50 \mathrm{~mol} \%$ TrFE. Introduction of VDF units may increase the dipole moment of main chain and increase $\Delta \varepsilon$ with increasing VDF content at lower VDF content. The amount of TrFE rich sequences would decrease at lower TrFE content than $50 \mathrm{~mol} \%$ since the mechanical $\alpha$ loss peak connected with molecular motion of TrFE rich sequences was depressed at a TrFE content below $50 \mathrm{~mol} \%{ }^{1}$. The amount of the defect arising from TrFE rich sequences decreases at lower TrFE content than 50 $\mathrm{mol} \%$. Thus. $\Delta \varepsilon$ due to the molecular motion of main chains with irregular and disordered conformation around the defects decreases at this lower TrFE content. Copolymers with TrFE content higher than $48 \mathrm{~mol} \%$ shows a crystal structure of the D

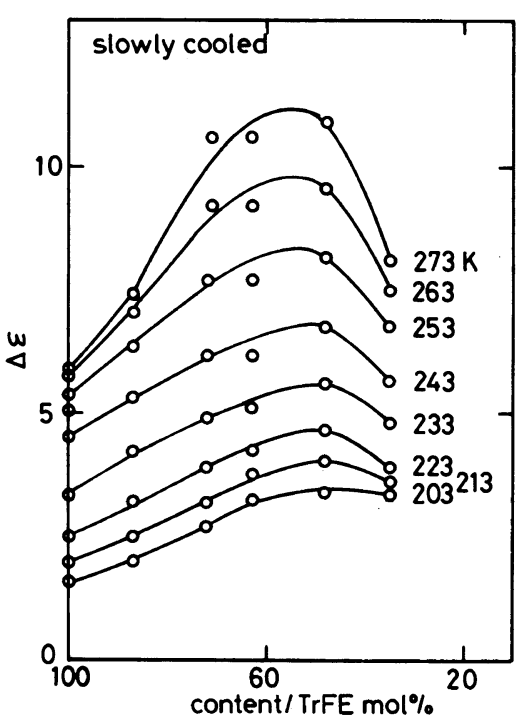

Figure 11. Plots of $\Delta \varepsilon$ against TrFE content for slowly cooled samples.

phase which is different from all-trans phase for copolymers with TrFE content lower than $35 \mathrm{~mol} \%$. This is discussed in the next section. Formation of the D phase may be related to amounts of TrFE rich sequence. The large values of $\Delta \varepsilon$ for 48 to $71 \mathrm{~mol} \%$ TrFE copolymers (samples 4, 6, and 8) reflect the high orientational polarizability of the molecular chains in the D phase. In VDF rich copolymers, rotational or torsional motion of main chains about the chain axis takes place in the crystalline region below $280 \mathrm{~K}$ and possibly contributes to the cooperative motion at the ferroelectric to paraelectric transition. ${ }^{19}$ The $\beta^{\prime}$ relaxation in the $D$ phase is substantially due to local molecular motion at lower temperature. However, the activation energy of this relaxation for sample 4 is similar to that of the molecular motion of the $T_{t}$ transition for copolymer with $48 \mathrm{~mol}^{\circ} \%$ TrFE. $^{10}$ Therefore the rotational molecular motion in the $\beta^{\prime}$ relaxation possibly contributes to the cooperative molecular motion at the $T_{\mathrm{t}}$ transition.

Crystal Structure of Slowly Cooled Samples

As observed in the previous study, ${ }^{11}$ slowly 
cooled copolymer samples with higher TrFE content above $48 \mathrm{~mol} \%$ exhibited double $D-E$ hysteresis. In a $j-E$ curve, two current peaks at $E_{1}$ and $E_{\mathrm{h}}$ appeared corresponding to the double hysteresis as shown for 48 and $63 \mathrm{~mol} \%$ TrFE copolymer (samples 4 and 6) in Figure 12. Figures 13(a), (b), and (c) show $j-E$ curves for sample 4 obtained by the following procedures: A high voltage triangular wave field was applied to a specimen for several cycles and the polarization and its reversal were repeated (a). When the triangular wave field is cut off at $A$ in (a) and started again from zero field, the $j-E$ curve is shown as (b) in the cycle. The curve of (c) is obtained if the field is cut off at B or $\mathrm{C}$ in (a). The current peak at $E_{\mathrm{h}}$ disappeared in Figure 13(b) while it remained in Figure 13(c). Considering the $j-E$ and $D-E$ curvves in Figures 12 and 13, the peak at $E_{\mathrm{h}}$ is due to a polarization process and the peak at $E_{1}$, to a depolarization process. X-Ray diffraction studies under an electric field in previous studies revealed that $\mathrm{X}$-ray patterns at $18-20^{\circ}$ in $2 \theta$ changed from single to double peak when the field increased to field $E_{1}$ and the pattern changed from a double to single peak

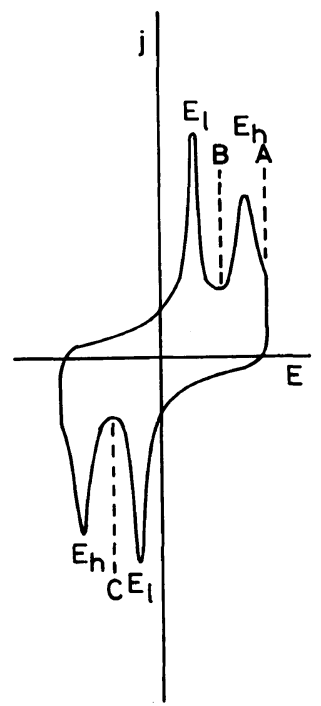

(a)
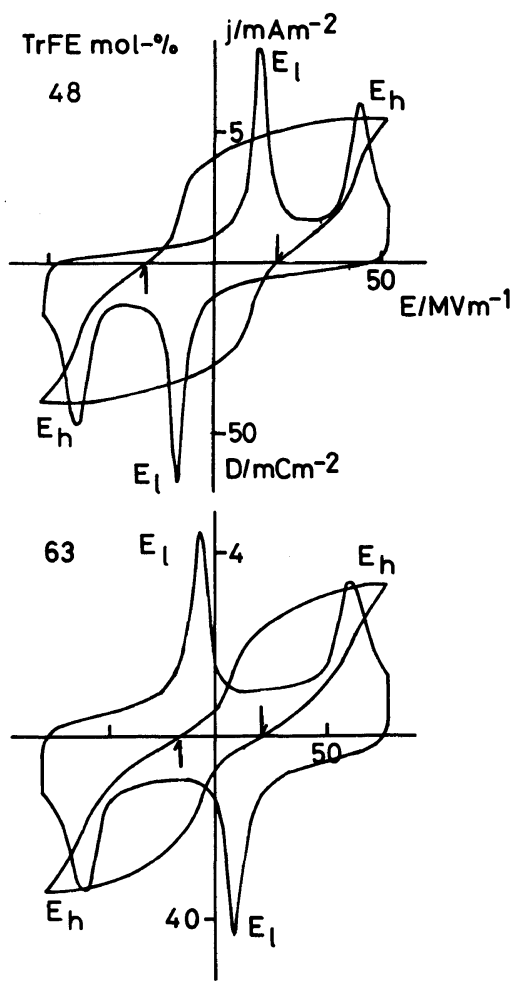

Figure 12. The $D-E$ and $j-E$ curves for slowly cooled copolymers with 48 and $63 \mathrm{~mol} \%$ TrFE (samples 4 and 6).

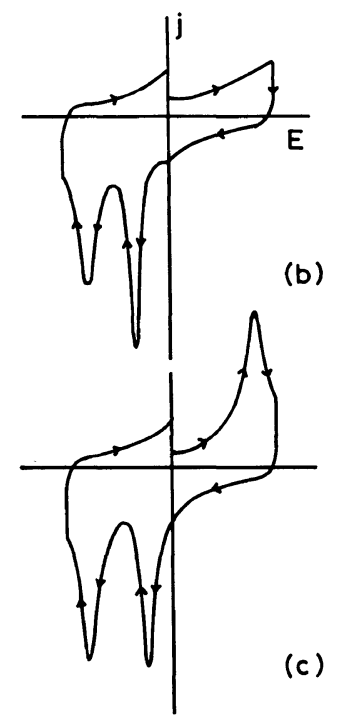

Figure 13. Changes in $j-E$ curves under different electric field conditions. (a): triangular wave field was applied repeatedly. (b) and (c): the field is cut off at A and B or C in (a), respectively. 
when the field further increased to $E_{\mathrm{h}} \cdot{ }^{11-13}$ These results were found in the repeated cycles of a high electric filed. Thus, the X-ray pattern gave a single peak at $A$ and a double peak at $B$ or $C$ in Figure 13(a). Unpoled slowly cooled samples also showed the X-ray double peak at $18-20^{\circ}$ in $2 \theta .^{1}$ Two different crystal structures were proposed for the X-ray double peak: an all-trans structure containing skew bonds proposed by Tashiro et al. ${ }^{19,20}$ and mixtures of disordered all-trans and 3/1 helix by Lovinger et al. ${ }^{21}$ and Horiuchi et al. ${ }^{22}$ The arrows in Figure 12 indicate the electric filed in the $j-E$ curves corresponding to the almost zero remanent polarization in the $D-E$ curves where the X-ray pattern shows a double peak. It is difficult to interpret such a polarization process by Tashiro's and Lovinger's structure models. Considering the data on density, IR, $\mathrm{X}$-ray and electric measurements, we propose crystal structures of the $T_{3} G_{3} \bar{G}$ conformation of main chains packed parallel and antiparallel for single and double X-ray peaks referred to as $S$ and $D$ phases, respectively. ${ }^{12,13}$ $\mathrm{X}$-Ray diffraction patterns from 33 to $43^{\circ}$ in $2 \theta$ are shown for poled and unpoled samples in Figure 14. The poled D phases were obtained by subjecting the slowly cooled samples to a high voltage triangular wave field for several cycles and the field was cut off at B or C in Figure 13(a). The X-ray patterns at 33 to $43^{\circ}$ in $2 \theta$ for unpoled samples are consistent with those for the poled D phases. Thus, the crystal structure of the unpoled samples seems to be the same as that of the poled D phases. A transformation from mixtures of all-trans and 3/1 helix to all-trans structures took place for specimens slightly oriented and poled by a high dc field. ${ }^{21}$ The transformation of structure from the D to all-trans phase did not take place for unoriented slowly cooled copolymer samples with $48 \mathrm{~mol} \%$ TrFE used under the poling conditions in this work. If a higher electric field or copolymers with TrFE content lower than $48 \mathrm{~mol} \%$ are used, the transformation from the $\mathrm{D}$ phase to all-trans phase

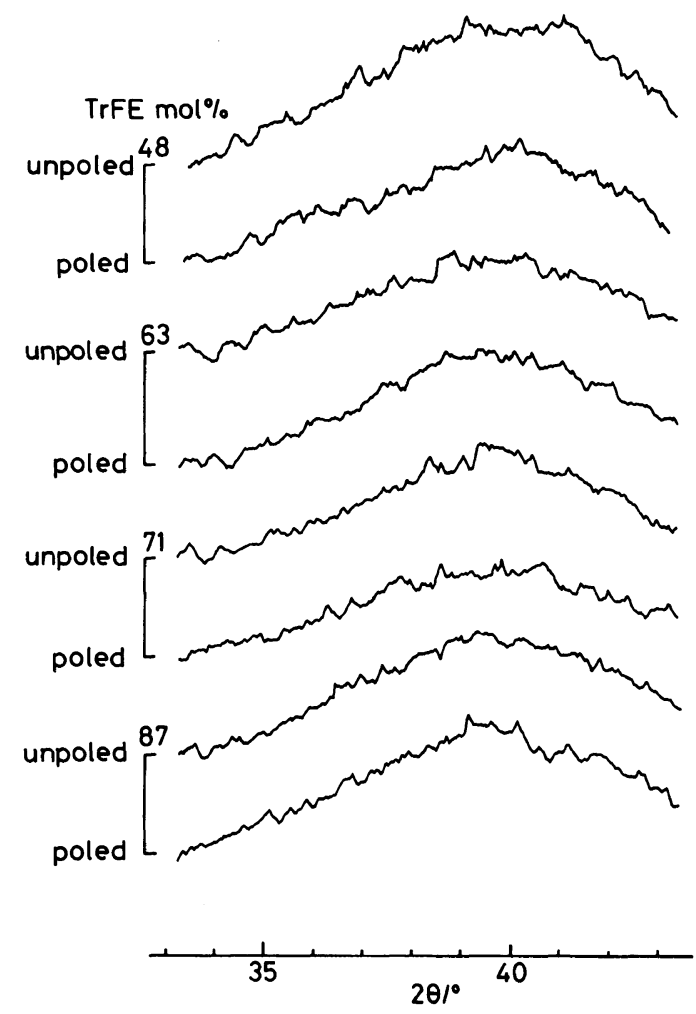

Figure 14. X-Ray diffraction patterns from 33 to $43^{\circ}$ in $2 \theta$ for poled and unpoled slowly cooled samples with 48 to $87 \mathrm{~mol} \%$ TrFE.

occurs. ${ }^{23}$ A slowly cooled sample of the copolymer with $35 \mathrm{~mol}^{\%}$ TrFE showed a single peak in $j-E$ curve. ${ }^{23}$ The crystal structure of this copolymer is well known to be of the alltrans phase since the $(001)$ reflection is at $35.5^{\circ}$ in $2 \theta .^{24}$

\section{High Temperature Transition}

The copolymer with high VDF content which took the all-trans phase showed a peak of dielectric constant $\varepsilon^{\prime}$ at ferroelectric to paraelectric phase transition $T_{\mathrm{t}}{ }^{10}$ The $\varepsilon^{\prime}$ peak was observed at 333 to $338 \mathrm{~K}$ also for copolymers with 48 to $87 \mathrm{~mol} \%$ TrFE as shown in the previous paper. ${ }^{11,25}$ The reciprocal of $\varepsilon^{\prime}$ is plotted against temperature for 48 to $87 \mathrm{~mol} \%$ TrFE copolymers in Figure 15 were $1 / \varepsilon^{\prime}$ decreases and increases almost linearly with increasing temperature for 48 to $87 \mathrm{~mol} \% \mathrm{TrFE}$ 


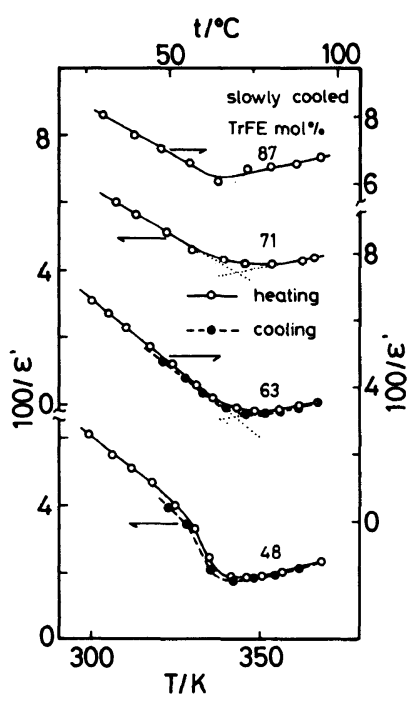

Figure 15. Plots of $1 / \varepsilon^{\prime}$ against temperature for slowly cooled samples with 48 to $87 \mathrm{~mol} \%$ TrFE. Solid line: heating, broken line: cooling.

copolymers in the vicinity of the transition $T_{t}$. The intersect of the two straight lines of plots of $1 / \varepsilon^{\prime} v s$. $T$ was taken as the transition temperature. Variation of $1 / \varepsilon^{\prime}$ with temperature $T$ was expressed by the Curie-Weiss relation

$$
\begin{array}{ll}
1 / \varepsilon^{\prime}=\left(T_{\mathrm{t}}-T\right) / C_{1}, & T<T_{\mathrm{t}} \\
1 / \varepsilon^{\prime}=\left(T-T_{\mathrm{t}}\right) / C_{2}, & T>T_{\mathrm{t}}
\end{array}
$$

Where $C_{1}$ and $C_{2}$ are empirical constants. The values of $T_{t}, C_{1}$ and $C_{2}$ are listed for slowly cooled copolymers with 63 and $71 \mathrm{~mol} \%$ TrFE in Table II. The values of $C_{1}$ and $C_{2}$ for these samples were larger than those for copolymer with $48 \mathrm{~mol}^{\circ} \% \operatorname{TrFE}^{4,8}$ The $T_{\mathrm{t}}$ transition in the copolymers with higher TrFE content was ascribed to the phase transition between ferroelectric and paraelectric phases by dielectric $^{3,4}$, dynamic mechanical ${ }^{1,2}$ and $\mathrm{X}-$ ray $^{20}$ studies. However, the D phase in slowly cooled samples exhibited the double $D-E$ hysteresis and had a very small value of remanent polarization of almost zero. Thus, the $T_{\mathrm{t}}$ transition is considered due to the phase transition between the $\mathrm{D}$ and paraelectric phases. The $\mathrm{D}$ phase for slowly cooled copolymers with high TrFE
Table II. Empirical constants $C_{1}$ and $C_{2}$ in the Curie-Weiss relation for copolymers with 63 and $71 \mathrm{~mol} \%$ TrFE

\begin{tabular}{crr}
\hline $\operatorname{TrFE}$ content $/ \mathrm{mol} \%$ & \multicolumn{1}{c}{63} & \multicolumn{1}{c}{71} \\
\hline$T_{\mathrm{t}} / \mathrm{K}$ & 345 & 344 \\
$C_{1} / \mathrm{K}$ & 1225 & 1714 \\
$C_{2} / \mathrm{K}$ & 4000 & 5172 \\
\hline
\end{tabular}

content exhibits the nature of antiferroelectrics in contrast to that of ferroelectrics for the copolymer with high VDF content. Variation of $1 / \varepsilon^{\prime}$ with temperature for 63 to $87 \mathrm{~mol} \%$ TrFE copolymers indicates the second order transition while an abrupt decrease in $1 / \varepsilon^{\prime}$ just below $T_{\mathrm{t}}$ for $48 \mathrm{~mol} \%$ TrFE copolymer is characteristic of the first order transition in Figure $15{ }^{26}$ The solid and broken lines of $1 / \varepsilon^{\prime}$ vs. $T$ curves in Figure 15 show heating and cooling processes, respectively. Thermal hysteresis of the transition was very small in copolymers with higher TrFE content in contrast to the large hysteresis of copolymers with $65 \mathrm{~mol} \%$ VDF. DTA curves in the temperature region near the $T_{\mathrm{t}}$ transition are shown for copolymers with 48 to $87 \mathrm{~mol} \%$ TrFE in Figure 16. Endothermic peaks were observed for copolymer with lower TrFE content than $48 \mathrm{~mol} \%$, indicating the first order transition. ${ }^{4}$ No peak was found for copolymers with 63 to $87 \mathrm{~mol} \%$ TrFE but an abrupt change in the base line like that of the glass transition known as the second order phase transition was observed in a temperature range from 313 to $359 \mathrm{~K}$. The change of the base line was larger for slowly cooled samples than that for quenched samples and took place near the $T_{\mathrm{t}}$ transition so that this change of the base line seems to be connected to the $T_{\mathrm{t}}$ transition in crystalline regions. However, linear expansion vs. temperature curves of 63 and $71 \mathrm{~mol} \%$ TrFE copolymers in Figures 17 and 18 showed a rather sudden change similar to that of 48 and $35 \mathrm{~mol} \%$ TrFE copolymers, indicating the first order of transition. ${ }^{27}$ The order of the $T_{\mathrm{t}}$ transition is complicated for 63 and $71 \mathrm{~mol} \%$ 


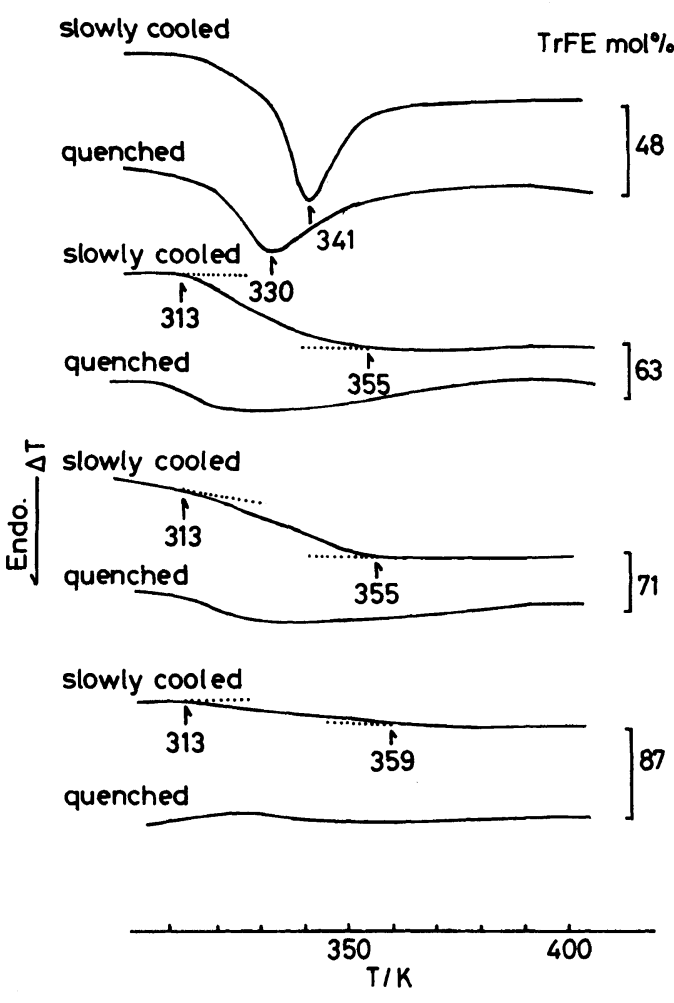

Figure 16. DTA curves in a temperature region of $T_{\mathrm{t}}$ transition for copolymers with 48 to $87 \mathrm{~mol} \%$ TrFE.

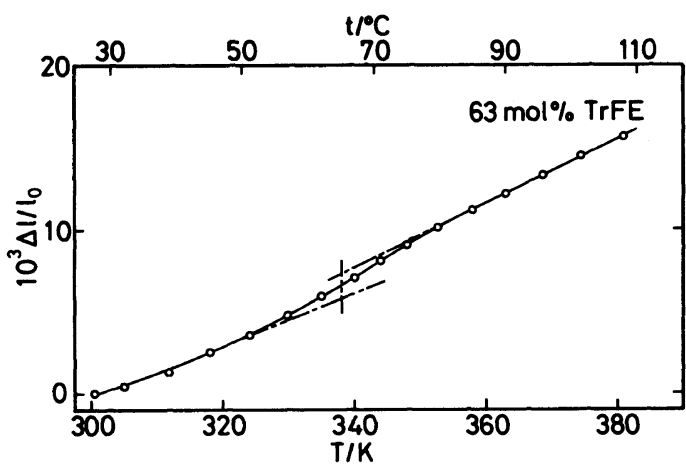

Figure 17. Plots of linear expansion against temperature for slowly cooled sample with $63 \mathrm{~mol} \%$ TrFE.

TrFE copolymers like that ${ }^{4,28}$ of the $48 \mathrm{~mol}^{\circ} \%$ TrFE copolymer. Pressure dependence of the $T_{t}$ transition was investigated by dielectric measurements. The plots of $1 / \varepsilon^{\prime} v s$. temperature are shown for slowly cooled 63 and $71 \mathrm{~mol} \%$ TrFE copolymers (samples 6 and 8) under

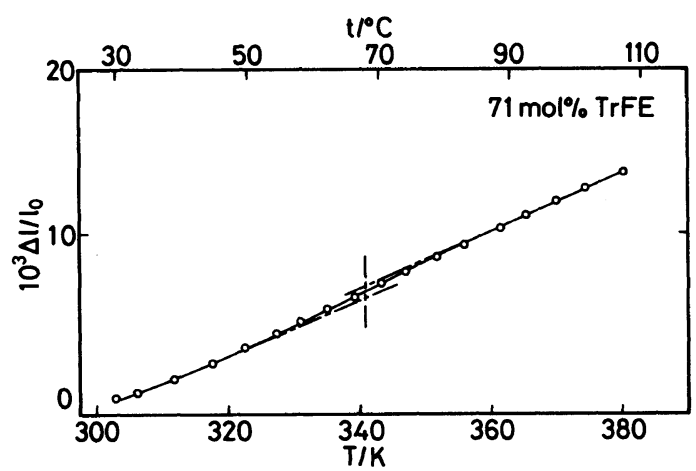

Figure 18. The same as Figure 17 for slowly cooled sample with $71 \mathrm{~mol} \%$ TrFE.

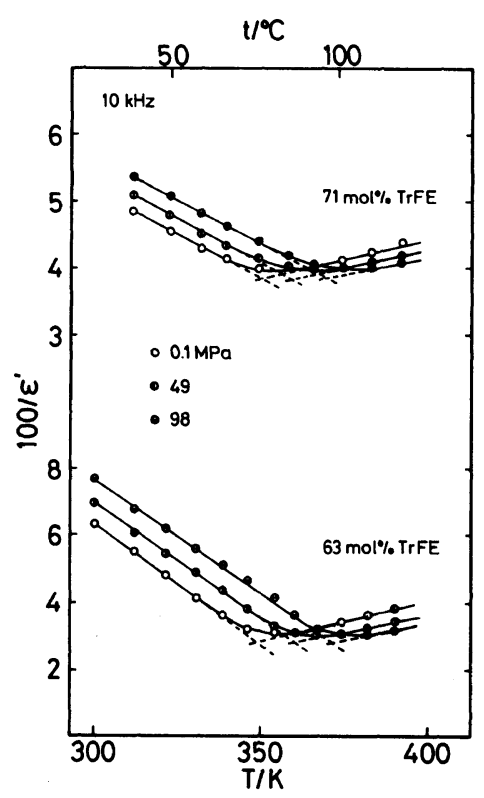

Figure 19. The same as Figure 15 at various pressures for slowly cooled samples with 63 and $71 \mathrm{~mol} \%$ TrFE (samples 6 and 8).

different pressure in Figure 19. The dependence of transition temperature on pressure was estimated at 0.19 and $0.22 \mathrm{~K} \mathrm{MPa}^{-1}$ for 63 and $71 \mathrm{~mol} \%$ TrFE copolymers, being smaller than 35 and $48 \mathrm{~mol}^{\%}$ TrFE copolymers. ${ }^{4,27}$ As $\operatorname{TrFE}$ content increased, the $\varepsilon^{\prime}$ peak at the $T_{\mathrm{t}}$ transition became smaller and the properties related to the transition became unclear as indicated by smaller changes in DTA base line and the liner expansion. These findings in- 
dicate that $T_{3} G_{3} \bar{G}$ conformation of main chains in crystals becomes disordered with increase of TrFE content since the remanent polarization in the $S$ phase estimated from $j-E$ curves for PTrFE and $87 \mathrm{~mol} \%$ copolymer was much smaller than that estimated from such a conformation.

\section{CONCLUSIONS}

Dielectric behavior of the VDF-TrFE copolymers with 35 to $87 \mathrm{~mol} \%$ TrFE was investigated over a wide range of frequency and temperature. The $\beta^{\prime}$ and $\beta$ relaxations and $T_{\mathrm{t}}$ transition were observed in increasing order of temperature. The $\beta^{\prime}$ relaxation was related to the molecular motion around the defects in crystals due to the anomalous linkages such as head-to-phead and tail-to-tail in TrFE rich sequences. The $\beta$ relaxation was attributed to micro-Brownian motion of the VDF rich sequences for 35 to $63 \mathrm{~mol} \%$ TrFE copolymers and was considered to consist of two components, $\beta_{\mathrm{a}}$ and $\beta_{\mathrm{c}}$, due to the local mode of molecular motions in the amorphous region and reorientation of main chains around the chain end defects in crystals for 71 and $83 \mathrm{~mol} \%$ TrFE copolymers. The $T_{\mathrm{t}}$ transition was connected with the phase transition between the D and paraelectric phases for 48 to $87 \mathrm{~mol} \%$ TrFE copolymers. The remanent polarization in the $\mathrm{D}$ phase was nearly zero like that of antiferroelectrics. The pressure dependence of the $T_{\mathrm{t}}$ transition was also observed for 63 and $71 \mathrm{~mol} \%$ TrFE copolymers and was smaller than that for copolymer with $48 \mathrm{~mol}^{\circ} \%$ TrFE. The $T_{\mathrm{t}}$ transition showed the characters of first or second order of transition which depended on the observed properties such as linear expansion, thermal and dielectric behavior. It was inferred that the molecular motion of the $\beta^{\prime}$ relaxation is closely related to that at the $T_{\mathrm{t}}$ transition.

Acknowledgements. The author wishes to thank Professor Emeritus N. Koizumi of
Kyoto University for his many valuable comments and suggestions for this work. Thanks are also due to Dr. Y. Kubouchi and Mr. S. Koizumi of Daikin Industries, Ltd., Osaka for providing samples.

\section{REFERENCES}

1. Y. Murata and N. Koizumi, Polym. J., 17, 385 (1985).

2. T. Yagi, Polym. J., 11, 353 (1979).

3. T. Furukawa, M. Ohuchi, A. Chiba, and M. Date, Macromolecules, 17, 1384 (1984).

4. N. Koizumi, N. Haikawa, and H. Habuka, Ferroelectrics, 57, 99 (1984).

5. Y. Murata, Polym. J., 20, 251 (1988).

6. A. Lovinger, T. Furukawa, G. T. Davis, and M. G. Broadhust, Polymer, 24, 1225 (1983).

7. K. Tashiro, K. Takano, M. Kobayashi, Y. Chatani, and H. Tadokoro, Polymer, 22, 1312 (1981).

8. T. Furukawa, M. Date, E. Fukada, Y. Tajitsu, and A. Chiba, Jpn. J. Appl. Phys., 19, L109 (1980).

9. H. Ohigashi and K. Koga, Jpn. J. Appl. Phys., 21, L455 (1982).

10. K. Kimura and H. Ohigasi, Appl. Phys. Lett., 43, 834 (1983).

11. N. Koizumi, Y. Murata, and T. Tsunashima, IEEE Trans., Electr. Insul., EI-21, 543 (1986).

12. Y. Oka, Y. Murata, and N. Koizumi, Polym. J., 18, 417 (1986).

13. Y. Oka, N. Koizumi, and Y. Murata, J. Polym. Sci., Polym. Phys. Ed., 24, 2059 (1986).

14. N. Koizumi, Y. Murata, and Y. Oka, Jpn. J. Appl. Phys., 23, L324 (1984).

15. Y. Murata, J. Hagino, and N. Koizumi, Kobunshi Ronbunshu, 36, 697 (1979).

16. G. Williams and D. C. Watts, Trans. Faraday Soc., 66, 80 (1970).

17. S. Yano, J. Polym. Sci. Part A 2, 9, 585 (1971).

18. Y. Murata, unpublished data.

19. K. Tashiro, K. Takano, M. Kobayashi, Y. Chatani, and H. Tadokoro, Polymer, 25, 195 (1984).

20. K. Tashiro, K. Takano, M. Kobayashi, Y. Chatani, and H. Tadokoro, Ferroelectrics, 57, 297 (1984).

21. A. J. Lovinger, G. T. Davis, T. Furukawa, and M. G. Broadhust, Macromolecules, 15, 323 (1982).

22. H. Horiuchi, K. Matsusige, and T. Takemura, Jpn.J. Appl. Phys., 25, L465 (1986).

23. N. Koizumi and Y. Murata, Ferroelectrics, in press.

24. A. J. Lovinger, T. Furukawa, G. T. Davis, and M. G. Broadhust, Polymer, 24, 1225 (1983).

25. Y. Higashihata, J. Sako, and T. Yagi, Ferroelectrics, 32, 85 (1981).

26. T. Mitui, I. Tatsuzaki, and E. Nakamura, "An Introduction to Physics of Ferroelectrics," Gordon 


\section{Relaxation and Transition of VDF-TrFE Copolymers}

and Breach Science, New York, 1976.

27. N. Koizumi, Y. Murata, and N. Haikawa, Jpn. J. Appl. Phys., 24, 862 (1985).
28. H. Kominami, K. Koyama, S. Ikeda, and Y. Wada, Rep. Prog. Polym. Phys. Jpn., 30, 429 (1987). 